\title{
Torsion-Free Abelian Semigroup Rings X
}

\author{
RYÛKI MATSUDA*
}

Let $R$ be a commutative ring, and let $S$ be a submonoid of a torsion-free abelian (additive) group (Then $S$ is called a g-monoid). We condider the semigroup ring $R[S]$ of $S$ over $R$. The aim of $\S 1$ of this note is to prove two Theorems on seminormality of $R[S]$. The aim of $\S 2$ of this note is to show that an MCsemigroup need not be a Macaulay semigroup. Torsion-free abelian semigroup rings IX is [M1].

\section{$\S 1$ Seminormal Semigroup Rings.}

We have the following results.

Theorem 1([AA]). Let $D$ be an integral domain. Then $D$ is seminormal and $S$ is seminormal if and only if $D[S]$ is seminormal.

$D$ is called seminormal if $\alpha^{2} \in D$ and $\alpha^{3} \in D$ imply $\alpha \in D$ for all $\alpha \in q(D)$ $(q(D)$ denotes the quotient field of $D) . S$ is called seminormal if $2 \alpha \in S$ and $3 \alpha \in S$ imply $\alpha \in S$ for all $\alpha \in q(S)(q(S)$ denotes the quotient group of $S$; $\left.q(S)=\left\{s-s^{\prime} \mid s, s^{\prime} \in S\right\}\right)$.

Theorem 2([M4, Theorem 3]). D is u-closed if and only if $D[S]$ is u-closed.

$D$ is called u-closed if $\alpha^{2}-\alpha \in D$ and $\alpha^{3}-\alpha^{2} \in D$ imply $\alpha \in D$ for all $\alpha \in q(D)$.

Proposition(cf. [OSY]). If $D$ is t-closed, then $D\left[X_{1}, X_{1}^{-1}, \cdots, X_{n}, X_{n}^{-1}\right]$ is $t$ closed, where $X_{1}, \cdots, X_{n}$ are indetermanates.

$D$ is called t-closed if $\alpha^{2}-a \alpha \in D$ and $\alpha^{3}-a \alpha^{2} \in D$ imply $\alpha \in D$ for all $a \in D$ and $\alpha \in q(D)$.

Lemma 1. Let $G$ be a torsion-free abelian group. Then $D$ is $t$-closed if and only if $D[G]$ is $t$-closed.

Proof. The sufficiency is straightforward.

The necessity: Assume that $F^{2}-f F \in D[G]$ and $F^{3}-f F^{2} \in D[G]$ for elements $f \in D[G]$ and $F \in q(D[G])$. There exists a finitely generated subgroup $H$ of $G$ such that $f \in D[H], F \in q(D[H]), F^{2}-f F \in D[H]$ and $F^{3}-f F^{2} \in D[H]$. $D[H]$ is isomorphic onto $D\left[X_{1}, X_{1}^{-1}, \cdots, X_{n}, X_{n}^{-1}\right]$ for some $n$. By Proposition, we have $F \in D[H]$.

Received June 1, 2000.

1991 Mathematics Subject Classification. Primary 13A15, Secondary 20M14.

* Department of Mathematical Sciences, Ibaraki University, Mito, Ibaraki 310-8512, Japan. 
Lemma 1 implies the folowing,

Lemma 2. $D$ is t-closed and $K[S]$ is $t$-closed if and only if $D[S]$ is $t$-closed, where $K=q(D)$.

Let $n$ be a positive integer $>1$. The following conditions are equivalent:

(1) $n \alpha,(n+1) \alpha, \cdots \in S$ imply $\alpha \in S$ for all $\alpha \in q(S)$.

(2) $S$ is seminormal.

Example 1. Example of $D[S]$ which is u-closed and which is not seminormal: Let $n$ be a positive integer $>1, S=\{0, n, n+1, n+2, \cdots\}$, and let $D$ be a u-closed domain. Then $D[S]$ is u-closed and $D[S]$ is not seminormal.

Example 2. Assume that $q(S)$ has torsion-free rank one.

(1) $D$ is seminormal and $S$ is integrally closed if and only if $D[S]$ is seminormal.

(2) $D$ is t-closed and $S$ is integrally closed if and only if $D[S]$ is t-closed.

A g-monoid $S$ is called integrally closed if $n \alpha \in S$ implies $\alpha \in S$ for all positive integers $n$ and $\alpha \in q(S)$.

Let $D$ be a domain, and let $S$ be a g-monoid. First, we consider what are conditions for the ring $D[S]$ to satisfy in order to $D$ being t-closed and $S$ being seminormal. Our result is the following,

Theorem 3. The following conditions are equivalent for the domain $D$ and the g-monoid $S$ :

(1) $D$ is t-closed and $S$ is seminormal.

(2) $F^{2}-a F \in D[S]$ and $F^{3}-a F^{2} \in D[S]$ imply $F \in D[S]$ for all elements $a \in D$ and $F \in q(D[S])$.

Condition (2) is clearly satisfied if $D[S]$ is t-closed.

Secondly, we consider to extend Theorem 1 for a commutative ring $R$ with zero-divisors.

$R$ is called seminormal if $\alpha^{2} \in R$ and $\alpha^{3} \in R$ imply $\alpha \in R$ for all $\alpha \in q(R)$ $(q(R)$ denotes the total quotient ring of $R ; q(R)=\{a / b \mid a \in R$ and $b$ is a nonzerodivisor of $R$ ). $R$ is called a von Neumann regular ring if, for each elemenrt $a$ of $R$, there exists an element $b$ of $R$ such that $a=a^{2} b$.

We have the following results.

Theorem 4([M5, Theorem 20]). Assume that $q(R)$ is a von Neumann regular ring. Then $R$ is integrally closed and $S$ is integrally closed if and only if $R[S]$ is integrally closed.

Theorem 5([BCM]). Assume that $q(R)$ is a von Neumann regular ring. If $R$ is seminormal, then $R[X]$ is seminormal.

Our result is the following,

Theorem 6. Assume that $q(R)$ is a von Neumann regular ring. Then $R$ is seminormal and $S$ is seminormal if and only if $R[S]$ is seminormal.

Proof of Theorem 3. Assume that condition (2) holds. 
Assume that $\alpha^{2}-a \alpha \in D$ and $\alpha^{3}-a \alpha^{2} \in D$ for elements $a \in D$ and $\alpha \in K$, where $K$ is the quotient field of $D$. By condition (2), we have $\alpha \in D[S]$, and hence $\alpha \in D$. Therefore $D$ is t-closed.

The condition (2) implies that $D[S]$ is seminormal. Then $S$ is seminormal by Theorem 1.

Next, assume that condition (1) holds. Then $D[S]$ is seminormal by Theorem 1. Let $G$ be the quotient group of $S$. Then $D[G]$ is t-closed by Proposition.

Assume that $F^{2}-a F \in D[S]$ and $F^{3}-a F^{2} \in D[S]$ for elements $a \in D$ and $F \in q(D[S])$. Since $D[G]$ is t-closed, we have $F \in D[G]$.

The case $a=0$ : Since $D[S]$ is seminormal, we have $F \in D[S]$.

The case $a \neq 0$ : We have $(F / a)^{2}-F / a \in K[S]$ and $(F / a)^{3}-(F / a)^{2} \in K[S]$, where $K=q(D) . K[S]$ is u-closed by Theorem 2. It follows that $F / a \in K[S]$, and hence $F \in K[S]$. Since $F \in D[G]$, we have $F \in D[S]$.

Therefore condition (2) holds.

Proof of Theorem 6. The sufficiency is straightforward.

The necessity: Let $X_{1}, X_{2}, X_{3}, \cdots$ be indeterminates. $R\left[X_{1}\right]$ is seminormal by Theorem 5. Suppose that $R\left[X_{1}, \cdots, X_{k-1}\right]$ is seminormal. $q\left(R\left[X_{1}, \cdots, X_{k-1}\right]\right)$ is a von Neumann regular ring by [M5, Lemma 17]. Therefore $\left(R\left[X_{1}, \cdots, X_{k-1}\right]\right)$ $\left[X_{k}\right]$ is seminormal. It follows that $R\left[X_{1}, X_{1}^{-1}, \cdots, X_{k}, X_{k}^{-1}\right]$ is seminormal for any $k$.

Assume that $F^{2} \in R[G]$ and $F^{3} \in R[G]$ for $F \in q(R[G])$. There exists a finitely generated subgroup $H$ of $G$ such that $F^{2} \in R[H], F^{3} \in R[H]$ and $F \in$ $q(R[H]) . R[H]$ is isomorphic onto $R\left[X_{1}, X_{1}^{-1}, \cdots, X_{n}, X_{n}^{-1}\right]$ for some $n$. Since $R\left[X_{1}, X_{1}^{-1}, \cdots, X_{n}, X_{n}^{-1}\right]$ is seminormal, we have $F \in R[H] \subset R[G]$. Therefore $R[G]$ is seminormal. We note that $q(R[H])$ is canonically regarded as a subring of $q(R[G])$.

Let $F^{2} \in R[S]$ and $F^{3} \in R[S]$ for $F \in q(R[S])$. Then $F \in R[G]$. Put $F=\sum a_{i} X^{\alpha_{i}}$, where $0 \neq a_{i} \in R$ and $\alpha_{i} \neq \alpha_{j}$ for $i \neq j$. Suppose that $\alpha_{k} \notin S$ for some $k$. Since $R$ has no non-zero nilpotents, there exists a prime ideal $P$ of $R$ which does not contain $a_{k}$. Set $D=R / P$. Then, in $D[G]$, we have $\bar{F}^{2} \in$ $D[S], \bar{F}^{3} \in D[S]$ and $\bar{F} \notin q(D)[S]$. This contradicts to Theorem 1. Therefore $R[S]$ is seminormal.

\section{§. An MC-semigroup need not be a Macaulay semigroup.}

In [SM1] we proved a semigroup version of Chapters 1, 2 and 3 of Narita's Ideal Theory [N]. In [SM3] we proved almost all propositions in Chapters 4 and 5 of $[\mathrm{N}]$ for g-monoids.

Let $I$ be an ideal of $S$, and $s \in S$. If $s+a \in I$ implies $a \in I$, then $s$ is called a non-zerodivisor on $S$ modulo $I$. If $s$ is not a non-zerodivisor on $S$ modulo $I$, then $s$ is called a zerodivisor. The set of zerodivisors on $S$ modulo $I$ is denoted by $\mathrm{Z}(S / I)$.

The ordered sequence $x_{1}, \cdots, x_{n}$ of elements of $S$ is called a regular sequence if $\left(x_{1}, \cdots, x_{n}\right) \varsubsetneqq S$ and if $x_{2} \notin Z\left(S /\left(x_{1}\right)\right), \cdots, x_{n} \notin Z\left(S /\left(x_{1}, \cdots, x_{n-1}\right)\right)$.

The maximum of lengths of all regular sequences in $I$ on $S$ is called the grade of $I$ on $S$, and is denoted by $\mathrm{G}(I, S)$. 
If $S$ is a Noetherian semigroup with maximal ideal $M$, and if $\mathrm{G}(M, S)$ equals the dimension of $S$, then $S$ is called a Macaulay semigroup([M3]).

Now, let $S$ be a Noetherian semigroup, and $I$ an ideal of $S$. If all prime divisors of $I$ have the same height, then $I$ is called unmixed.

Let $S$ be a Noetherian semigroup. Assume that, if $I$ is an ideal of $S$ which is generated by the number height $(I)$ of elements, then $I$ is unmixed. Then $S$ is called an MC-semigroup.

In [SM3] we posed the following,

Problem. Is a g-monoid $S$ a Macaulay semigroup if and only if $S$ is an MCsemigroup?

It is well-known that the corresponding notions are equivalent for Noetherian rings.

The aim of this section is to answer to the Problem.

Lemma 1(A part of [M3, Theorem 4]). Let $S$ be a Noetherian semigroup, and let $x_{1}, \cdots, x_{n}$ be a regular sequence on $S$. Then any permutation of the $x$ 's is a regular sequence on $S$.

Lemma 2([M2, Theorem 3]). In an integrally closed Noetherian semigroup $S$, each prime divisor of any proper principal ideal has height one.

Example([SM2]). Let $x_{1}+x_{2}=x_{3}+x_{4}$ be a unique relation of letters $x_{1}, x_{2}, x_{3}$ and $x_{4}$. Set $S=\mathbf{Z}_{0} x_{1}+\mathbf{Z}_{0} x_{2}+\mathbf{Z}_{0} x_{3}+\mathbf{Z}_{0} x_{4}$, where $\mathbf{Z}_{0}$ is the non-negative integers. Then $S$ is a g-monoid with maximal ideal $M=\left(x_{1}, x_{2}, x_{3}, x_{4}\right)$. Set $Q_{1}=\left(x_{2}, x_{3}, x_{4}\right), Q_{2}=\left(x_{1}, x_{3}, x_{4}\right), Q_{3}=\left(x_{1}, x_{2}, x_{4}\right), Q_{4}=\left(x_{1}, x_{2}, x_{3}\right), P_{1}=$ $\left(x_{1}, x_{3}\right), P_{2}=\left(x_{1}, x_{4}\right), P_{3}=\left(x_{2}, x_{3}\right)$ and $P_{4}=\left(x_{2}, x_{4}\right)$. Then $Q_{i}$ and $P_{i}$ are prime ideals of $S$ for each $i$. There do not exist other prime ideals of $S$ than $M, Q_{1}, Q_{2}, Q_{3}, Q_{4}, P_{1}, P_{2}, P_{3}$ and $P_{4}$. It follows that $S$ is a Noetherian semigroup of dimension three, and $M$ is not a minimal prime divisor of any ideal generated by three elements.

We note that, if $M$ is a maximal ideal of a two-dimensional Noetherian semigroup, then $M$ is a minimal prime divisor of an ideal generated by two elements ([M2, Theorem 5]).

We will show that the g-monoid $S$ of our Example is not a Macaulay semigroup and that $S$ is an MC-semigroup. Throughout the section, $S$ denotes the g-monoid in Example.

Lemma 3. (1) Let $l_{1}, l_{2}, l_{3}$ be integers. Then $l_{1} x_{1}+l_{2} x_{2}+l_{3} x_{3}=0$ if and only if $l_{1}=l_{2}=l_{3}=0$.

(2) Let $a_{1}, a_{2}, a_{3}, a_{4}, b_{1}, b_{2}, b_{3}, b_{4}$ be non-negative integers. Then

$a_{1} x_{1}+a_{2} x_{2}+a_{3} x_{3}+a_{4} x_{4}=b_{1} x_{1}+b_{2} x_{2}+b_{3} x_{3}+b_{4} x_{4}$

if and only if

$b_{1}-a_{1}=b_{2}-a_{2}=a_{3}-b_{3}=a_{4}-b_{4}$.

Lemma 4. $S$ is integrally closed.

Proof. Let $\alpha=l_{1} x_{1}+l_{2} x_{2}+l_{3} x_{3}$ be an element of the quotient group $\mathrm{q}(S)$ of $S$ with $l_{i} \in \mathbf{Z}$. We will determine conditions for $\alpha \in S$. 
We have $l_{1} x_{1}+l_{2} x_{2}+l_{3} x_{3}=k_{1} x_{1}+k_{2} x_{2}+k_{3} x_{3}+k_{4} x_{4}$ with $k_{i} \geq 0$. By Lemma 3 , we have $l_{1}=k_{1}+k_{4}, l_{2}=k_{2}+k_{4}$ and $l_{3}=k_{3}-k_{4}$. Hence $k_{4} \leq$ $\min \left(l_{1}, l_{2}\right)$ and $l_{3} \geq-k_{4} \geq-\min \left(l_{1}, l_{2}\right)$. That is, $l_{1} \geq 0, l_{2} \geq 0$ and $l_{3} \geq-\min$ $\left(l_{1}, l_{2}\right)$.

If $\alpha=l_{1} x_{1}+l_{2} x_{2}+l_{3} x_{3}$ and $n \alpha \in S$ with $n>0$, then $n l_{1} \geq 0, n l_{2} \geq 0$ and $n l_{3} \geq-\min \left(n l_{1}, n l_{2}\right)$. Hence $\alpha \in S$. That is, $S$ is integrally closed.

Lemmas 2 and 4 imply that each principal ideal $I$ of $S$ is unmixed.

Proiposition 1. $S$ is not a Macaulay semigroup.

Proof. Suppose that $f_{1}, f_{2}, f_{3}$ is a regular sequence on $S$. We must derive a contradiction. We may assume that $f_{1}$ is of the form $x_{1}+g_{1}$ with $g_{1} \in S$. Then $x_{1}, f_{2}, f_{3}$ is a regular sequence on $S$. We use Lemma 1 . Then $f_{2}, x_{1}, f_{3}$ is a regular sequence. Suppose that $f_{2}$ is of the form $x_{3}+g_{2}$ with $g_{2} \in S$. Then $x_{3}, x_{1}, f_{3}$ is a regular sequence. But $x_{1}+x_{2} \in\left(x_{3}\right)$ and $x_{2} \notin\left(x_{3}\right)$; a contradiction. Hence $f_{2}$ is of the form $x_{2}+g_{4}$ with $g_{4} \in S$. Then $x_{2}, x_{1}, f_{3}$ is a regular sequence. Thus we have a contradiction.

Lemma 5. Let I be an ideal of $S$ with height two which is generated by two elements. Then $I$ is of the form $\left(n x_{1}, m x_{2}\right)$ or $\left(n x_{3}, m x_{4}\right)$ with positive integers $n$ and $m$.

Proof. Let $f_{1}=a_{1} x_{1}+a_{2} x_{2}+a_{3} x_{3}+a_{4} x_{4}$ and $f_{2}=b_{1} x_{1}+b_{2} x_{2}+b_{3} x_{3}+b_{4} x_{4}$ be generators for $I$ with non-negative integers $a_{1}, a_{2}, a_{3}, a_{4}, b_{1}, b_{2}, b_{3}$ and $b_{4}$.

Assume that $a_{1}>0$. Since $I$ is not contained in any of $P_{1}, P_{2}, P_{3}$ and $P_{4}$, we have $b_{1}=b_{3}=b_{4}=0$. Hence $I$ is of the form $\left(n x_{1}, m x_{2}\right)$.

The other cases are similar.

Lemma 6. Let $n$ and $m$ be positive integers. Then we have,

(1) The ideal $n P_{1} \cup m P_{3}$ is an $Q_{4}$-primary ideal.

(2) $n P_{2} \cup m P_{4}$ is an $Q_{3}$-primary ideal.

Proof. Let $A \in Q_{4}$ and $B \in M-Q_{4}$, and assume that $A+B \in n P_{1} \cup m P_{3}$. It is sufficient to show that $A \in n P_{1} \cup m P_{3}$.

$A$ is of the form $c_{1} x_{1}+c_{2} x_{2}+c_{3} x_{3}+c_{4} x_{4}$ where the $c_{i}$ are non-negative integers, $B$ is of the form $p x_{4}$ with $p>0, A+B$ is of the form

(1) $a x_{1}+a^{\prime} x_{3}+a_{1} x_{1}+a_{2} x_{2}+a_{3} x_{3}+a_{4} x_{4}$

or

(2) $b x_{2}+b^{\prime} x_{3}+b_{1} x_{1}+b_{2} x_{2}+b_{3} x_{3}+b_{4} x_{4}$,

where $a, a^{\prime}, a_{1}, a_{2}, a_{3}, a_{4}, b, b^{\prime}, b_{1}, b_{2}, b_{3}$ and $b_{4}$ are non-negative integers with $a+a^{\prime}=n$ and $b+b^{\prime}=m$.

Case (1):

$A+B=c_{1} x_{1}+c_{2} x_{2}+c_{3} x_{3}+\left(c_{4}+p\right) x_{4}=\left(a+a_{1}\right) x_{1}+a_{2} x_{2}+\left(a^{\prime}+a_{3}\right) x_{3}+a_{4} x_{4}$.

By Lemma 3, there exists an integer $l$ such that

$c_{1}=a+a_{1}+l, c_{2}=a_{2}+l, c_{3}=a^{\prime}+a_{3}-l$

and

$c_{4}+p=a_{4}-l$.

Since $c_{1}+c_{3} \geq n$, we have $A \in n P_{1}$. 
Case (2): The similar proof shows that $A \in m P_{3}$.

The proof is complete.

Lemma 7. Let $n$ and $m$ be positive integers. Then we have,

(1) $\left(n x_{1}, m x_{2}\right)=\left(n P_{1} \cup m P_{3}\right) \cap\left(n P_{2} \cup m P_{4}\right)$.

(2) $\left(n x_{3}, m x_{4}\right)=\left(n P_{1} \cup m P_{2}\right) \cap\left(n P_{3} \cup m P_{4}\right)$.

Proof. It is sufficient to show that each element $x$ of $\left(n P_{1} \cup m P_{3}\right) \cap\left(n P_{2} \cup m P_{4}\right)$ belongs to $I=\left(n x_{1}, m x_{2}\right)$.

Each element of $n P_{1} \cup m P_{3}$ is of the form

$A=a x_{1}+a^{\prime} x_{3}+a_{1} x_{1}+a_{2} x_{2}+a_{3} x_{3}+a_{4} x_{4}$

or

$B=b x_{2}+b^{\prime} x_{3}+b_{1} x_{1}+b_{2} x_{2}+b_{3} x_{3}+b_{4} x_{4}$

where $a, a^{\prime}, a_{1}, a_{2}, a_{3}, a_{4}, b, b^{\prime}, b_{1}, b_{2}, b_{3}$ and $b_{4}$ are non-negative integers with $a+a^{\prime}=n$ and $b+b^{\prime}=m$.

Similarly, each element of $n P_{2} \cup m P_{4}$ is of the form

$C=c x_{1}+c^{\prime} x_{4}+c_{1} x_{1}+c_{2} x_{2}+c_{3} x_{3}+c_{4} x_{4}$

or

$D=d x_{2}+d^{\prime} x_{4}+d_{1} x_{1}+d_{2} x_{2}+d_{3} x_{3}+d_{4} x_{4}$,

where $c, c^{\prime}, c_{1}, c_{2}, c_{3}, c_{4}, d, d^{\prime}, d_{1}, d_{2}, d_{3}$ and $d_{4}$ are non-negative integers with $c+c^{\prime}=n$ and $d+d^{\prime}=m$.

There arise four cases: (1) $x=A=C$, (2) $x=A=D$, (3) $x=B=C$ and (4) $x=B=D$.

Case (1):

$x=\left(a+a_{1}\right) x_{1}+a_{2} x_{2}+\left(a^{\prime}+a_{3}\right) x_{3}+a_{4} x_{4}=\left(c+c_{1}\right) x_{1}+c_{2} x_{2}+c_{3} x_{3}+\left(c^{\prime}+c_{4}\right) x_{4}$.

By Lemma 3 , there exists an integer $l$ such that

$c+c_{1}=a+a_{1}+l, c_{2}=a_{2}+l, c_{3}=a^{\prime}+a_{3}-l$ and $c^{\prime}+c_{4}=a_{4}-l$.

If $a^{\prime}+a_{3} \leq a_{4}$, then

$x=\left(a+a_{1}\right) x_{1}+a_{2} x_{2}+\left(a^{\prime}+a_{3}\right)\left(x_{3}+x_{4}\right)+\left(a_{4}-a^{\prime}-a_{3}\right) x_{4}=\left(a+a_{1}\right) x_{1}+$ $a_{2} x_{2}+\left(a^{\prime}+a_{3}\right)\left(x_{1}+x_{2}\right)+\left(a_{4}-a^{\prime}-a_{3}\right) x_{4} \in\left(n x_{1}\right)$.

If $a^{\prime}+a_{3} \geq a_{4}$, then $c_{3} \geq c^{\prime}+c_{4}$.

$x=\left(c+c_{1}\right) x_{1}+c_{2} x_{2}+\left(c_{3}-c^{\prime}-c_{4}\right) x_{3}+\left(c^{\prime}+c_{4}\right)\left(x_{3}+x_{4}\right)=\left(c+c_{1}\right) x_{1}+$ $c_{2} x_{2}+\left(c_{3}-c^{\prime}-c_{4}\right) x_{3}+\left(c^{\prime}+c_{4}\right)\left(x_{1}+x_{2}\right) \in\left(n x_{1}\right)$.

Hence $x \in I$.

Case (2):

$x=\left(a+a_{1}\right) x_{1}+a_{2} x_{2}+\left(a^{\prime}+a_{3}\right) x_{3}+a_{4} x_{4}=d_{1} x_{1}+\left(d+d_{2}\right) x_{2}+d_{3} x_{3}+\left(d^{\prime}+d_{4}\right) x_{4}$.

By Lemma 3 , there exists an integer $l$ such that

$d_{1}=a+a_{1}+l, d+d_{2}=a_{2}+l, d_{3}=a^{\prime}+a_{3}-l$

and $d^{\prime}+d_{4}=a_{4}-l$.

If $a^{\prime}+a_{3} \leq a_{4}$, then

$x=\left(a+a_{1}\right) x_{1}+a_{2} x_{2}+\left(a^{\prime}+a_{3}\right)\left(x_{3}+x_{4}\right)+\left(a_{4}-a^{\prime}-a_{3}\right) x_{4}=\left(a+a_{1}\right) x_{1}+$ $a_{2} x_{2}+\left(a^{\prime}+a_{3}\right)\left(x_{1}+x_{2}\right)+\left(a_{4}-a^{\prime}-a_{3}\right) x_{4} \in\left(n x_{1}\right)$.

If $a^{\prime}+a_{3} \geq a_{4}$, then $d^{\prime}+d_{4} \leq d_{3}$.

$x=d_{1} x_{1}+\left(d+d_{2}\right) x_{2}+\left(d_{3}-d^{\prime}-d_{4}\right) x_{3}+\left(d^{\prime}+d_{4}\right)\left(x_{3}+x_{4}\right)=d_{1} x_{1}+(d+$ $\left.d_{2}\right) x_{2}+\left(d_{3}-d^{\prime}-d_{4}\right) x_{3}+\left(d^{\prime}+d_{4}\right)\left(x_{1}+x_{2}\right) \in\left(m x_{2}\right)$.

Hence $x \in I$.

Cases (3) and (4) are similar. 
Proposition 2. $S$ is an MC-semigroup.

Proof. Let $I$ be an ideal of $S$ which is generated by the number ht $(I)$ of elements. We must show that $I$ is unmixed.

The case of $\operatorname{ht}(I)=1$ : Then $I$ is unmixed by Lemmas 2 and 4 .

The case of ht $(I)=2$ : Then $I$ is of the form $\left(n x_{1}, m x_{2}\right)$ or $\left(n x_{3}, m x_{4}\right)$ with positive integers $n$ and $m$ by Lemma 5 .

If $I=\left(n x_{1}, m x_{2}\right)$, then $I=\left(n P_{1} \cup m P_{3}\right) \cap\left(n P_{2} \cup m P_{4}\right)$ by Lemma 7. $n P_{1} \cup m P_{3}$ is $Q_{4}$-primary, and $n P_{2} \cup m P_{4}$ is $Q_{3}$-primary by Lemma 6 . Since $\operatorname{ht}\left(Q_{3}\right)=$ $\operatorname{ht}\left(Q_{4}\right)=2, I$ is unmixed.

If $I=\left(n x_{3}, m x_{4}\right)$, the proof is similar.

The case of ht $(I)=3$ : Example shows that the case can not happen.

We have proved that an MC-semigroup need not be a Macaulay semigroup.

\section{REFERENCES}

[AA] D. D. Anderson and D. F. Anderson, Divisorial ideals and invertible ideals, J. Algebra 76 (1982), 549-569.

[BCM] J. Brewer,D. Costa and K. McCrimmon, Seminormality and root closure in polynomial rings, J. Algebra 58 (1979), 217-226.

[M1] R. Matsuda, Torsion-free abelian semigroup rings IX, Bull. Fac. Sci., Ibaraki Univ. 26 (1994), 1-12.

[M2] R. Matsuda, Some theorems for semigroups, Math. J. Ibaraki Univ. 30 (1998), 1-7.

(M3] R. Matsuda, Note on Macaulay semigroups, Tsukuba J. Math. 23 (1999), 189-199.

[M4] R. Matsuda, Note on u-closed semigroup rings, Bull. Austral. Math. Soc. 59 (1999), 467-471.

[M5] R. Matsuda, Note on integral closures of semigroup rings, Tamkang J. Math. 31 (to appear).

[N] M. Narita, Introduction of Ideal Theory, Kyoritsu, 1970.

[OSY] N. Onoda,T. Sugatani and K. Yoshida, Local quasinormality and closedness type criteria, Houston J. Math. 11 (1985), 247-256.

[SM1] K. Satô and R. Matsuda, Note on Narita's Ideal Theory, Memoirs Tôhoku Inst. Tech. 19 (1999), 9-26.

[SM2] K. Satô and R. Matsuda, On 2-dimensional Noetherian semigroups and a principal ideal theorem, Math. J. Ibaraki Univ. 31 (1999), 29-31.

[SM3] K. Satô and R. Matsuda, Note on Narita's Ideal Theory, II, Memoirs Tôhoku Inst. Tech. (to appear.). 